\title{
Improvement of the performance of a $\mu$-metal magnetically shielded room by means of active compensation
}

\author{
H J M ter Brake, H J Wieringa and H Rogalla \\ University of Twente, Faculty of Applied Physics, Low Temperature Division, \\ PO Box 217, $7500 \mathrm{BE}$ Enschede, The Netheriands
}

Received 15 November 1990, accepted for publication 4 February 1991

\begin{abstract}
An active compensation technique is presented for improving the performance of a $\mu$-metal magnetically shielded room. Active compensation is established by measuring the magnetic field inside the room by a SQUiD magnetometer. The output of this sensor is amplified and connected to a coil surrounding the room. The magnetic field generated in this way compensates the measured field inside the room. Active compensation was tested for magnetic fields in the vertical direction on a shielded room with one $\mu$-metal shield. At low frequencies a shielding improvement of typically $40 \mathrm{~dB}$ was obtained. Measurements performed on a room with two $\mu$-metal shields indicated that the attainable improvement is not limited by the amount of $\mu$-metal applied in the room. The active compensation set-up is described in detail and experiments performed on the two magnetically shielded rooms are presented and discussed.
\end{abstract}

\section{Introduction}

The number of magnetically shielded rooms (MSRs) installed all over the world has grown extensively during the last few years. Only about four MSRs were in use in 1981, whereas the company Vacuumschmelze GmbH installed 38 rooms during the period 1985-1989 [1]. By far the largest application lies in the field of biomagnetism, where extremely weak magnetic fields originating from the human body are detected with SQUID sensors [2]. Although some researchers persist in trying to establish these measurements without an MSR, the world's leading groups on biomagnetism are all equipped with such rooms for reducing the impact of environmental noise.

However, one does not solve all the environmental noise problems simply by buying an MSR. Firstly, the low-frequency shielding is relatively poor, and secondly the MSR distorts the environmental noise fields. The latter effect creates gradients inside the room which are usually detected by biomagnetic equipment. A simple method to solve these problems is active compensation. Basically, this is established by means of a closed negative feedback loop consisting of a magnetic field sensor, control electronics and a more or less complicated coil set surrounding the MSR. The electronics process the output of the field sensor and control the current through the coil set. This is done in such a way that the magnetic field measured by the sensor is compensated.

The principle has been presented previously by Kelhä et al [3]. They used a fluxgate magnetometer outside their MSR as a sensor and, by incorporating a PID controller, established a shielding improvement of $35 \mathrm{~dB}$ at $0.1 \mathrm{~Hz}$ and $20 \mathrm{~dB}$ at $1 \mathrm{~Hz}$. Although these values depend on the position of the sensor, their approach can be applied to improve the performance of an MSR in general. However, when the MSR is used for biomagnetic experiments involving a multichannel SQUID system, it is better to apply a sQuID magnetometer as the field sensor. The resulting configuration is simpler: the MSR already functions as a low-pass filter. Furthermore, the shielding improvement is typically $40 \mathrm{~dB}$ for frequencies up to $1 \mathrm{~Hz}$, whereas the improvement does not critically depend on the sensor position. A further advantage of the latter method is that it can be applied to any MSR, no matter how many $\mu$-metal layers are used in the room. The only effect of more $\mu$ metal is a reduction of the loop gain, which can be easily compensated by extra amplification of the SQUID sensor output. The lowfrequency shielding improvement, therefore, can always be typically $40 \mathrm{~dB}$.

In this paper we first discuss the relevant limitations of an MSR, especially with regard to biomagnetism. After that, our active compensation approach will be described in detail, directly followed by measurements. Finally, the results will be discussed and major impacts will be considered.

\section{Limitations of a magnetically shlelded room}

The installation of an MSR affects in the first place the experimental set-up. In biomagnetic applications, for 
instance, the positioning of the subject with respect to the measuring equipment can be a problem; peripheral problems such as feedthroughs, ventilation and illumination may also arise. However, in this paper we focus on two limitations with respect to the actual noisereducing performance of an MSR: the relatively poor shielding for lower frequencies and the distortion of the environmental noise fields inside the room.

The walls of an MSR consist of a sandwich structure of a high-conductivity material (usually an aluminium alloy) and a high-permeability material (a nickel-iron alloy, usually called $\mu$-metal). The former establishes an eddy-current shielding, whose efficiency increases with the frequency - it starts to be effective above about $0.2 \mathrm{~Hz}$ in standard MSRs. The $\mu$ metal, however, gives a practically frequency-independent shielding for biomagnetic relevant frequencies (up to $100 \mathrm{~Hz}$ ). It more or less absorbs the field lines of the environmental noise field through its magnetization; that is, until the material is saturated. Our MSR (Vacuumschmelze type AK 2b [4]) has a shielding performance of $60 \mathrm{~dB}$ above $10 \mathrm{~Hz}$ but only $20 \mathrm{~dB}$ below $0.1 \mathrm{~Hz}$. This relatively poor shielding for lower frequencies is a problem because on the one hand, low-frequency signals may be relevant (see e.g. [5]), whereas on the other hand environmental noise has many low-frequency components. For instance, a car passing at a distance of $90 \mathrm{~m}$ with a speed of $50 \mathrm{~km} \mathrm{~h}^{-1}$, generates noise of roughly $2 \times 10^{-10} \mathrm{~T}$ showing a peak in the frequency spectrum at about $0.02 \mathrm{~Hz}$ [6]. Furthermore, the geomagnetic field fluctuations exhibit a $1 / f$ like behaviour with a spectral density up to $10^{-9} \mathrm{~T} \mathrm{~Hz}^{-1 / 2}$ at $0.1 \mathrm{~Hz}[7]$.

The effect of the field distortion can be understood by having a closer look at the biomagnetic sensing system. Usually, biomagnetic experiments are performed with gradiometers that measure field gradients. They should, in principle, be insensitive to uniform noise fields; however, due to limited accuracy and errors in the construction of such gradiometers, sensitivity to uniform fields inevitably arises. This sensitivity is called the imbalance of the gradiometer, represented by the factor $C_{\mathrm{b}}$. This imbalance factor $C_{\mathrm{b}}$ is equal to the ratio of the effectively measured field and the applied field. 'As-made' gradiometers have $C_{\mathrm{b}}$ values of $10^{-2}$ to $10^{-3}$; these values can be reduced to well below $10^{-4}$ by means of balancing techniques [8]. If such a gradiometer is placed inside an MSR the situation is much different. In this case, a uniform magnetic field is distorted by the walls and thus gradients arise inside the MSR which are detected by the gradiometer. This can be quantified as an effective contribution to the imbalance. We measured in our MSR, for a magnetic field in the vertical direction, $C_{\mathrm{b}}=3 \times 10^{-3}$ in the centre and $C_{b}=1.2 \times 10^{-2}$ at $60 \mathrm{~cm}$ above the floor in the centre (employing a gradiometer with a baseline of $5 \mathrm{~cm}$ ). The standard double $\mu$-metal walled MSRs have low-frequency shielding factors somewhat below 100 . This means that a reasonably balanced gradiometer is more sensitive to uniform environmental magnetic noise inside the MSR than outside it! Furthermore, one may conclude that it makes no sense at all to try to realize highly balanced gradiometers to be used inside an MSR. One way to improve this situation is to use more spherically shaped rooms - an ideal spherical MSR does not create these gradients. However, in the case of standard rectangularly shaped MSRs, active compensation can be used to reduce the effect of the environmental field distortion.

\section{Actlve compensation}

The idea of active compensation was described in the introduction. The basic scheme of such a compensation circuit is depicted in figure 1 . Here, the environmental noise source is represented by a current $I_{\mathrm{N}}$ through a coil. Via the transfer function $\beta_{\mathrm{NN}}$, it resuits in a noise field $B_{\mathrm{N}}$ or a noise gradient $G_{\mathrm{N}}$ at the position of the reference noise sensor. The output $V_{N}$ of this sensor, which follows via the sensor transfer function $H_{\mathrm{N}}$, is fed back with an amplification $A$ as a current $I_{\mathrm{C}}$ through the compensation coil. This is done in such a way that the contribution of $I_{\mathrm{C}}$ to $B_{\mathrm{N}}$ (or $G_{\mathrm{N}}$ ) via the transfer function $\beta_{\mathrm{CN}}$ compensates the environmental noise contribution. Thus, the reference noise signal $V_{\mathrm{N}}$ is reduced by a factor $1+H_{\mathrm{N}} A \beta_{\mathrm{CN}}$ following

$$
V_{\mathrm{N}}=H_{\mathrm{N}} I_{\mathrm{N}} \beta_{\mathrm{NN}} /\left(1+N A \beta_{\mathrm{CN}}\right) \text {. }
$$

The aim of course is to compensate for the contribution of $I_{\mathrm{N}}$ to the measuring signal $V_{\mathrm{S}}$. It can easily be derived that this signal is given by

$$
V_{\mathrm{S}}=H_{\mathrm{S}} I_{\mathrm{N}} \beta_{\mathrm{NS}}\left(\frac{1+H_{\mathrm{N}} A \beta_{\mathrm{CN}}-H_{\mathrm{N}} A \beta_{\mathrm{CS}} \beta_{\mathrm{NN}} / \beta_{\mathrm{NS}}}{1+H_{\mathrm{N}} A \beta_{\mathrm{CN}}}\right)
$$

including the compensation. Here, $\beta_{\mathrm{CS}}$ and $\beta_{\mathrm{NS}}$ represent the transfer functions from, respectively, $I_{\mathrm{C}}$ and $I_{\mathrm{N}}$ to the signal field $B_{\mathrm{S}}$ or signal gradient $G_{\mathrm{S}}$, whereas the signal-sensor transfer function is written as $H_{S}$. Optimum compensation is obtained if the signal $V_{\mathrm{S}}$ is zero, i.e. no environmental disturbances are present in the measuring signal. This leads to the condition

$$
\frac{\beta_{\mathrm{CN}}+1 / H_{\mathrm{N}} A}{\beta_{\mathrm{CS}}}=\frac{\beta_{\mathrm{NN}}}{\beta_{\mathrm{NS}}} \text {. }
$$

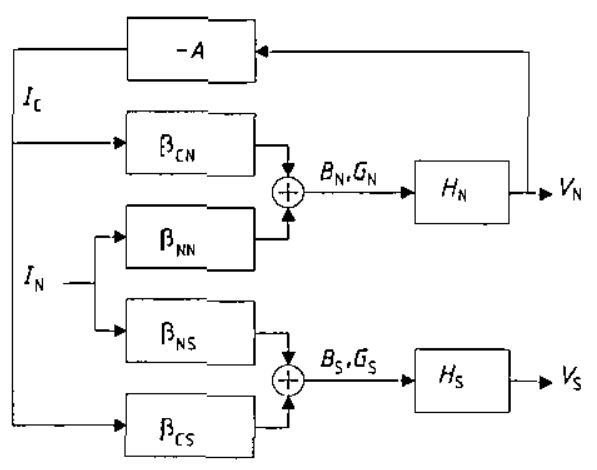

Figure 1. Basic scheme of the measuring set-up with active compensation. 
For large feedback gains $A$, this condition can be approximated by

$$
\beta_{\mathrm{CN}} / \beta_{\mathrm{CS}}=\beta_{\mathrm{NN}} / \beta_{\mathrm{NS}}
$$

According to equation (4), the ratio between the reference noise signal $V_{\mathrm{N}}$ and the measuring signal $V_{\mathrm{S}}$ due to the compensation current has to be the same as that due to the environmental noise. In that case, equation (2) shows that by compensation the contribution of the noise to the measuring signal is also reduced by the factor $1+H_{\mathrm{N}} A \beta_{\mathrm{CN}}$.

The above considerations are valid whether or not an MSR is incorporated, but let us first focus on the case without an MSR. If in that case both sensors are magnetometers (instead of gradiometers), compensation may work. However, interference from power lines will normally dominate $V_{\mathrm{N}}$. Therefore, either the compensation is designed to reduce the power-line interference, or this interference is filtered from $V_{N}$ to be able to compensate actively for relatively small low-frequency noise contributions. The former case was presented by Marzetta in 1961 [9]. In the latter case the filtering will introduce phase shifts in the compensation circuit so that not all frequency contributions will be compensated and, even more problematic, some may even be increased.

Active compensation without an MSR should, for biomagnetic experiments, be applicable to a gradiometer as the signal sensor. If the noise reference is a magnetometer, uniform noise-field contributions in the signal $V_{\mathrm{S}}$ (resulting from the imbalance of the gradiometer) can, in principle, be compensated. A problem may be that the compensation coil should generate a sufficiently uniform magnetic field. Furthermore, the above mentioned limitations due to power-line interference are also valid here. As a final case without an MSR, a gradiometer can be used as the noise reference sensor and an astatic Helmholtz coils set for providing a first-order gradient compensating field. Besides the power line problems, the alignment of gradiometers and coil set for establishing equation (3) will be very critical. To our knowledge active compensation without an MSR has not been used successfully in combination with biomagnetic experiments.

If an MSR is incorporated, the applicability of active compensation changes significantly. Firstly, power line interference is sufficiently shielded by the high-conductivity walls. Secondly, the environmental noise field, as well as the compensating field, is more or less condensed in the $\mu$-metal walls, especially for low frequencies. Therefore the uniformity of the compensation coil is not so critical as it is outside the MSR. Both advantages, of course, only hold if the noise-reference sensor as well as the signal-measuring sensor is inside the MSR. In this respect the approach of Kelhä et al, as discussed in the introduction, is not very obvious; because their reference sensor is outside the MSR, problems with regard to power line interference and alignment did arise. If, however, $V_{N}$ and $V_{\mathrm{S}}$ are obtained inside the MSR, the method is very promising especially if both sensors are magnetometers.

If instead $V_{\mathrm{S}}$ is a gradiometer output, the situation is more complicated. The gradiometer signal can be split into three contributions:

(1) the contribution of the residual field in the MSR due to the imbalance of the gradiometer;

(2) the contribution of the residual gradient in the MSR;

(3) the gradient contribution to the distortion of the uniform field by the $\mu$-metal walls.

These three contributions occur for both the noise field and the compensation field. If the noise-reference sensor is a magnetometer, the contributions (1) and (3), both linearly dependent on the environmontal ficld, cain be reduced and the signal in the gradiometer is determined by the residual gradients of the noise field and the compensation field. The application of a gradiometer as the noise-reference sensor inside the MSR is practically impossible because of a stability problem: field fluctuations with relatively high frequencies (above about $0.1 \mathrm{~Hz}$ ) are shielded by eddy currents in the highconductivity walls. These eddy currents generate gradients in the MSR that add to the three signal contributions stated above in the gradiometer. Normally, the eddycurrent contribution is opposite to the resultant signal of the other three contributions. This effect is illustrated in figure 2 where a negative step in the environmental field is applied. The response of a gradiometer directly after applying the step is positive, caused by the eddycurrent contribution. As the eddy currents decay $(\tau=$ $0.9 \mathrm{~s}$ ), the low-frequency contributions to the gradiometer signal show up as a negative signal. This means that if low-frequency noise is fed back in a negative loop, so as to establish active compensation, the highfrequency noise is fed back in a positive way, which may give rise to an unstable loop. A more complicated controller could deal with this non-minimum phase behaviour of the room and the gradiometer together, but then all transfer functions would have to be known in detail. Furthermore, these transfer functions depend on the position of the gradiometer with respect to the walls of the MSR.

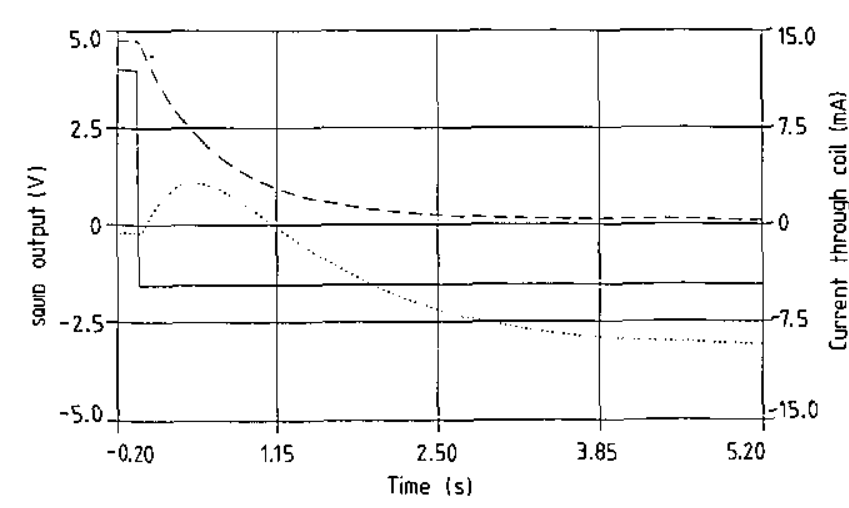

Figure 2. Responses of a gradiometer and a magnetometer inside the MSR (type AK 2b) to a step in the applied field at $t=0$ : full curve, current through the coil surrounding the MSR; broken curve, magnetometer output; dotted curve, gradiometer output. 


\section{Experiments}

Active compensation was tested on the shielded room at the Biomagnetic Centre of the University of Twente (Vacuumschmelze, type AK 2b [4]). It consists of an aluminium wall covered on the inside with one $\mu$-metal shield. The noise-reference sensor used was a 15 turn coil with a diameter of $1 \mathrm{~cm}$, which was coupled to a BTi RF SQUID system. The output of the SQUID system was amplified and then fed into an Amcron audio amplifier, which served as a proportional controller. This amplifier fed a current through two 11-turn coils which were wound around the bottom and the top of the MSR. The size of the coils was $4.8 \mathrm{~m} \times 3.4 \mathrm{~m}$ and the vertical separation between the coils was $2.3 \mathrm{~m}$. They were wound in the same direction and connected in series. This set of coils provided the compensating field in the vertical direction. A single coil surrounding the MSR was installed for generating disturbing fields. This coil consisted of 14 turns, measuring $6.7 \mathrm{~m} \times 4.6 \mathrm{~m}$. It was placed $90 \mathrm{~cm}$ above the centre of the room. The field inside the room was measured independently by a second field sensor consisting of a single-turn loop having a diameter of $1 \mathrm{~cm}$, also connected to a BTi RF SQUID system. This sensor can be considered as the signal sensor in the scheme of figure 1.

The operation of the compensating feedback loop is optimal if the loop gain is as high as possible (see equation (2)). The higher the gain, the higher the additional shielding will be. However, if the loop gain is set too high, the feedback loop will be unstable and will start to oscillate. A $100 \mathrm{~Hz}$ low-pass filter was used to prevent the amplifier from being saturated with highfrequency components, which do not contribute to the compensation due to phase shifts in the signal.

The transfer functions $\beta$ corresponding to figure 1 depend somewhat on the positions of the sensors with respect to the MSR. If both sensors are in the centre, $\beta_{\mathrm{CN}}=\beta_{\mathrm{CS}}$ and $\beta_{\mathrm{NN}}=\beta_{\mathrm{NS}}$. The relevant transfer functions for that case are presented in table 1. A low-frequency shielding factor of 12 is taken into account in the evaluation of the functions $\beta$.

The shielding of the room, with and without active compensation, is presented in figure 3 . The performance of the active compensation was measured by the signal sensor positioned at the centre of the room. The reference sensor was placed at various positions, i.e. at the centre of the room, at $60 \mathrm{~cm}$ from the centre in the longitudinal

Table 1. Low-frequency magnitude of transfer functions corresponding to figure 1 for the noise sensor and the signal sensor at the MSR centre.

\begin{tabular}{llll}
\hline Transfer function & Magnitude & Unit & Accuracy (\%) \\
\hline$A$ & 0.129 & $\mathrm{~A} \mathrm{~V}^{-1}$ & \pm 5 \\
$H_{\mathrm{N}}$ & $5.01 \times 10^{10}$ & $\mathrm{~V} \mathrm{~T}^{-1}$ & \pm 1 \\
$H_{S}$ & $7.2 \times 10^{7}$ & $\mathrm{~V} \mathrm{~T}^{-1}$ & \pm 10 \\
$\beta_{\mathrm{CN}}=\beta_{\mathrm{CS}}$ & $3.65 \times 10^{-7}$ & $\mathrm{~T} \mathrm{~A}^{-1}$ & \pm 2 \\
$\beta_{\mathrm{NN}}=\beta_{\mathrm{NS}}$ & $2.25 \times 10^{-7}$ & $\mathrm{~T} \mathrm{~A}^{-1}$ & \pm 2 \\
\hline
\end{tabular}

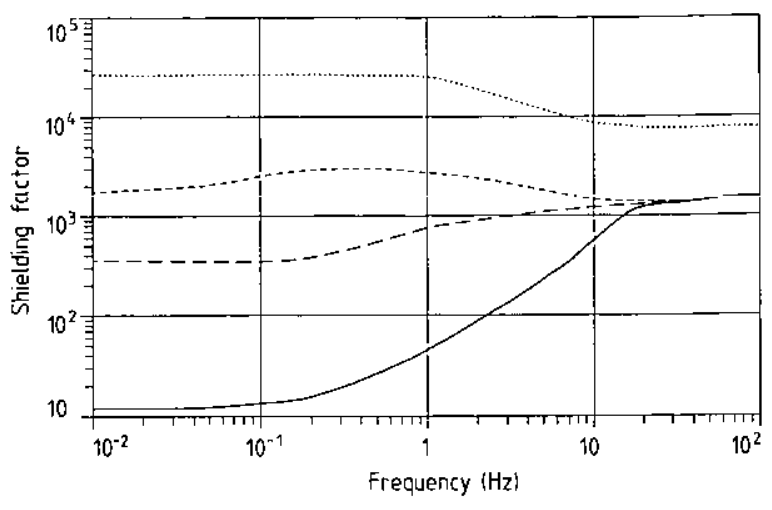

Figure 3. Shielding performance of MSR type AK 2b measured at the centre of the MSR: full curve, without active compensation; dotted curve, with active compensation, reference sensor at MSR centre; short broken curve, with active compensation, reference sensor at $60 \mathrm{~cm}$ from the centre in longitudinal (horizontal) direction (i.e. position $x$ ); long broken curve, with active compensation, reference sensor placed $40 \mathrm{~cm}$ below position $x$

(horizontal) direction (position $x$ ), and $40 \mathrm{~cm}$ below the latter position. Obviously, the best active compensation is obtained if the reference sensor is at the same position as the signal sensor. In that case the condition given by equation (4) is satisfied and the shielding is improved by a factor of $1+H_{\mathrm{N}} A \beta_{\mathrm{CN}}$. According to table 1 this improvement factor should be $2360( \pm 8 \%)$. For low frequencies the measured factor, according to figure 3, was $2250( \pm 5 \%)$, so the results are in very good agreement. For higher frequencies the improvement factor decreases because $\beta_{\mathrm{CN}}$ decreases. This is due to the increasing eddy-current shielding.

As a comparison, the shielding performance of a room with two $\mu$-metal shields was measured, and some preliminary results on active compensation were obtained. These measurements were performed at the Philips Research Laboratory in Hamburg (Germany), where a magnetically shielded room of type AK $3 a$ [4] from Vacuumschmelze was available. Figure 4 depicts

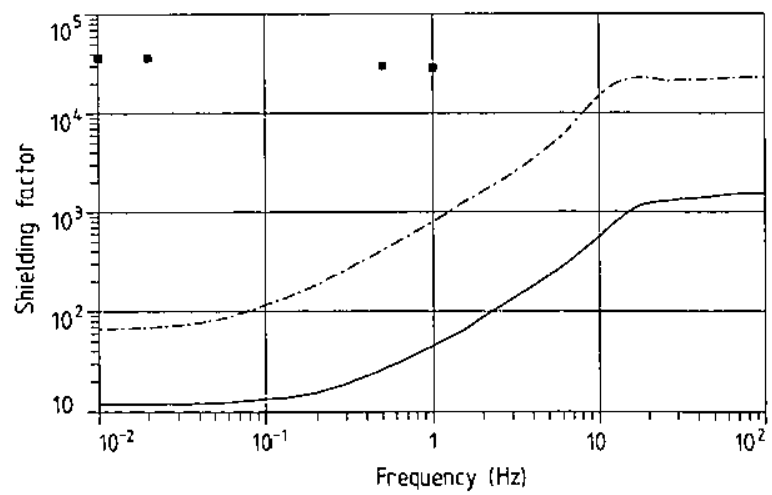

Figure 4. Shielding performance without active compensation of MSR type AK $3 a$ (chain curve) and MSR type AK 2b (full curve) measured at their centres. $\mathbf{a}$, shielding performance of AK $3 \mathrm{a}$ with active compensation, reference sensor at the MSR centre. 
the shielding factor as a function of frequency for this room. The shielding factor of our MSR is included in this figure. Clearly, the additional $\mu$-metal shield adds an extra shielding factor which is independent of the frequency. This additional shielding factor reduces the overall gain of the active-compensation feedback loop. Therefore the reference signal needs extra amplification for obtaining the same shielding improvement via active compensation as for our MSR. This amplification factor has to be the same as the additional attenuation of the magnetic field by the extra $\mu$-metal layer. In this way the total loop gain remains the same. Figure 4 also shows the data of some preliminary measurements on active compensation, applied to this AK 3a MSR. The total loop gain in this preliminary set-up was only 450 $( \pm 8 \%)$. The measured shielding improvement was 520 $( \pm 5 \%)$ so this is also in good agreement with theory.

In order to demonstrate that active compensation is not only effective for artificial disturbances, the field inside our room due to environmental disturbances was recorded for $5 \mathrm{~min}$. After $3 \mathrm{~min}$ the active compensation was switched on. The result is shown in figure 5 and is self-evident.

So far only magnetometers have been involved. However, biomagnetic measurements are often performed using gradiometers. We did some active compensation measurements on the three-channel system, which is currently in use at the Biomagnetic Centre at Twente. It consists of three first-order gradiometers, each with a diameter of $40 \mathrm{~mm}$ and a baseline length of $50 \mathrm{~mm}$. As an indication of the environmental magnetic field, the output of a fluxgate magnetometer was used - placed outside the MSR at several meters distance from the $\mu$ metal walls. To show the effect of active compensation, $10 \mathrm{~min}$ of environmental noise was recorded. Without active compensation the responses of the gradiometers exactly follow the low-frequency disturbances of the environmental field, the higher frequencies $(>0.1 \mathrm{~Hz})$

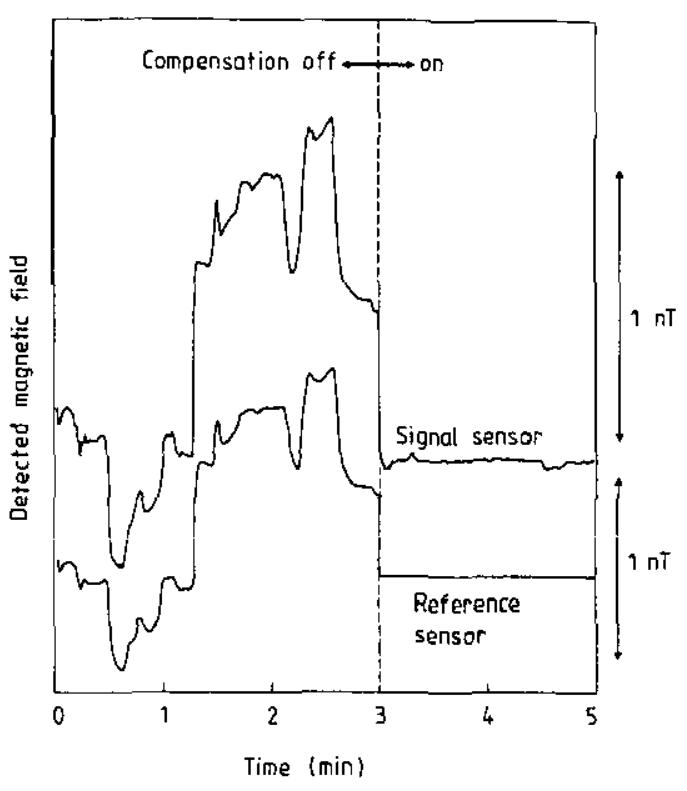

Figure 5. Effect of active compensation on environmentalfield fluctuations measured inside the MSR (type AK 2b): upper trace, output of the signal sensor at the MSR centre; lower trace, output of the noise reference sensor placed $40 \mathrm{~cm}$ below position $x$ (see text).

being relatively better shielded by the room. This is shown in figure 6. When active compensation is applied, the disturbances in the gradiometer signals are reduced by at least a factor of 5 (see figure 7). Obviously the improvement is less spectacular than it is for magnetometers. This is caused by the fact that the transfer function of the environmental field to the magnetometer is quite different from that of the environmental field to the gradiometer. Therefore, the condition as stated in equation (4) is not met equally well. Nevertheless an improvement by at least a factor of five is significant and useful.

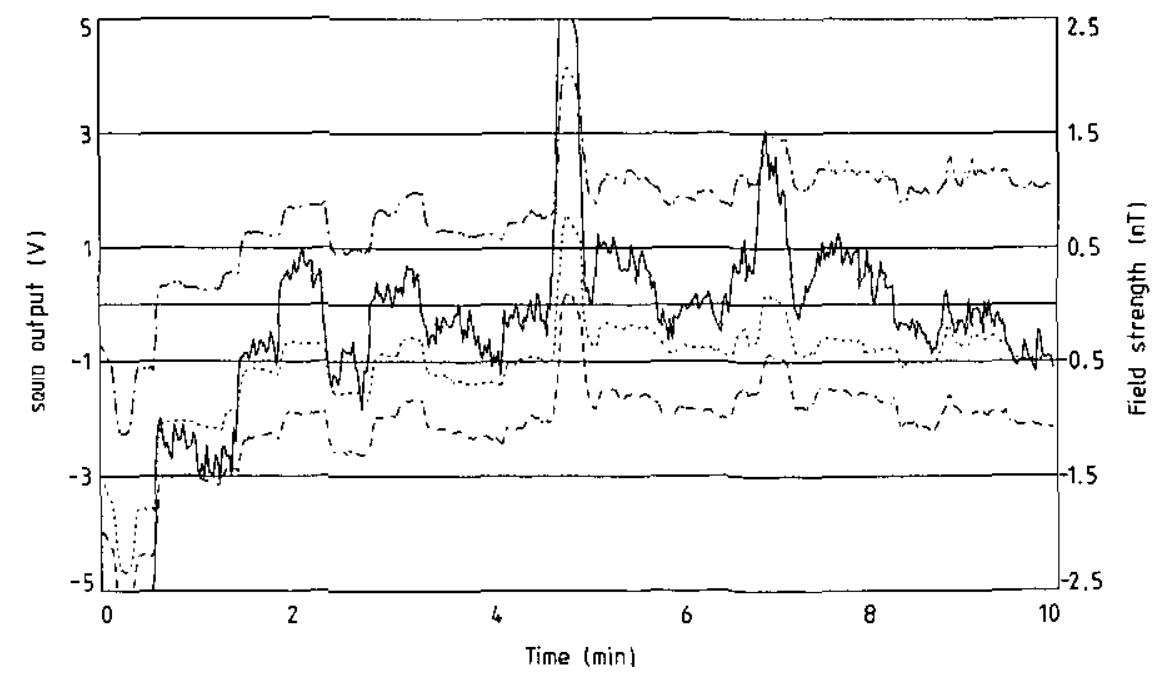

Figure 6. Environmental-field fluctuations measured by three first-order gradiometers at the MSR centre (type AK $2 b$, broken curves) and the magnetic field recorded outside the MSR with a fluxgate magnetometer (full curve); without active compensation. 


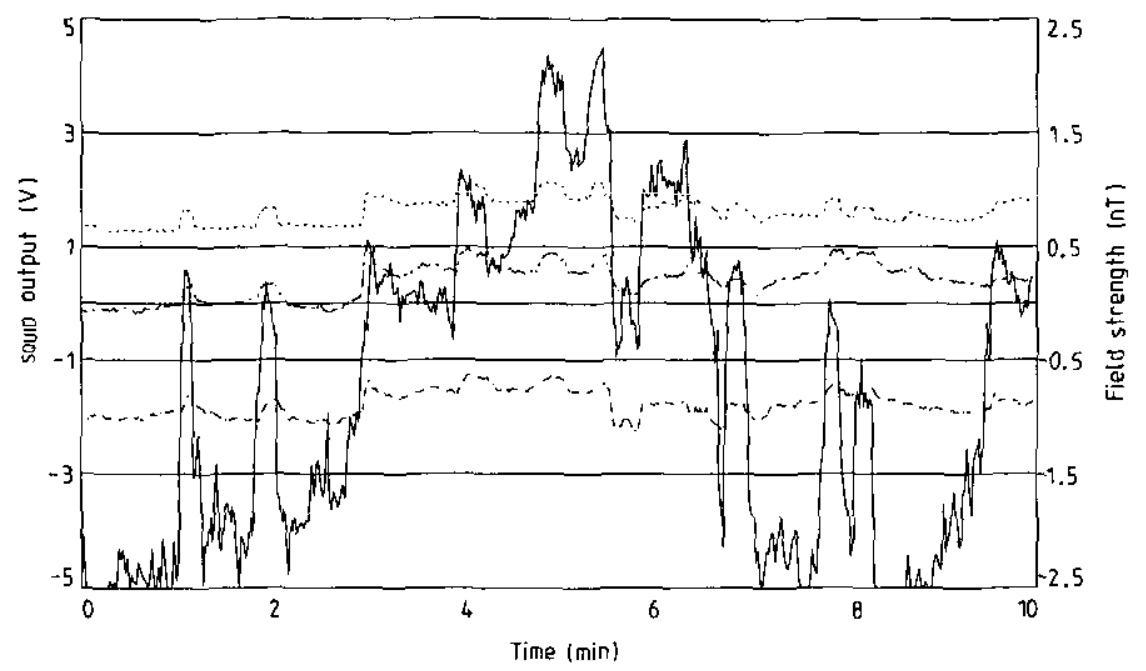

Figure 7. As figure 6 but with active compensation.

\section{Discussion}

Active compensation was tested on a MSR with one $\mu$ metal shield. A low-frequency shielding improvement of typically $40 \mathrm{~dB}$ can be established. Measurements performed on a room with two $\mu$-metal shields indicate that the shielding improvement via active compensation is not dependent on the amount of $\mu$ metal used for the room. More $\mu$ metal only requires extra amplification of the reference noise sensor output.

Active compensation is especially useful if magnetometers are used as signal sensors. If gradiometers are applied, environmental field fluctuations are not that dramatically reduced. We measured a reduction by a factor of at least 5 , which is useful but significantly smaller than the reduction of $40 \mathrm{~dB}$ for magnetometers. One should therefore consider the applicability of magnetometers (instead of gradiometers) as the biomagnetic sensors inside a magnetically shielded room with active compensation.

A remaining problem is that the environmental noise, in contrast to our applied noise field, is not restricted to the vertical direction. Therefore, a compensation merely in the vertical direction cannot compensate all disturbances. For that purpose the system of active compensation should be expanded to three orthogonal directions. Each direction needs its own reference sensor, compensation coil and feedback amplifier.

The controller that we used was a simple amplifier acting as a proportional controller. The compensation can be improved by using a proportional derivative controller. The derivative action of such a controller compensates for the integrative behaviour of the shielded room. It facilitates a further increase of the loop gain, thereby improving the performance of the active compensation.

\section{Acknowledgments}

We thank the biomagnetic/squid group of Philips, Hamburg, for their helpful discussions and for the opportunity to perform measurements on their shielded room. Further, we wish to thank $Z$ Dunajski and $R$ van Benthem for their assistance in performing the experiments at the Biomagnetic Centre, Twente, and J Mondt for critically reading the manuscript.

\section{References}

[1] Best K J Vacuumschmelze GmbH, Hanau, Germany Private communication

[2] Katila T E 1989 Advances in Biomagnetism ed S J Williamson et al (New York: Plenum) pp 19-32

[3] Kelhä V O, Pukki J M, Peltonen R S, Penttinen V J, Ilmoniemi $\mathrm{R} \mathrm{J}$ and Heino $\mathrm{J} \mathrm{J} 1982$ IEEE Trans. Magn. 18 260-70

[4] Specifications of Vacuumschmelze magnetically shielded rooms Vacuumschmelze GmbH company paper M 053 Walk-in magnetically shielded rooms

[5] Di Luzio S, Comani S, Romani G L, Basile M, Del Gratta C and Pizella V 1989 Nuovo Cimento 11 D $1853-9$

[6] Vrba J CTF Systems Inc., Canada Private communication

[7] Swithenby S J 1980 J. Phys. E: Sci. Instrum. 13 801-13

[8] Ter Brake H J M, Dunajski Z, Van der Mheen W A G and Flokstra J 1989 J. Phys. E. Sci. Instrum. 22 $560-4$

[9] Marzetta L A 1961 Rev. Sci. Instrum. 32 1192-5 